\title{
Costos ambientales en plantaciones de Pinus radiata D. Don*
}

\author{
Environmental costs in Pinus radiata D. Don. forest plantations
}

\section{JORGE GAYOSO}

Facultad de Ciencias Forestales, Universidad Austral de Chile, Casilla 567, Valdivia, Chile

\begin{abstract}
SUMMARY
This study proposes a quantitative environmental assessment method. Economic consequences related to forest operations in Pinus radiata D. Don forests, under the concept of soil and water sustainable natural capital were modeled. Environmental costs consider the costs of mitigation actions required to reduce impact levels up to the impacts caused by the use of the best available technology.

The model applies a standard costs matrix to an impact coefficient matrix according to the level of sensitivity of the soil. The result is valuation of environmental costs in monetary units per hectare. The model allows impartial assessment at company, regional and national levels.

Three scenarios and technologic appropriateness levels were simulated. Environmental costs range from 2246 US\$/ ha $\left(5.61 \mathrm{US} \$ / \mathrm{m}^{3}\right)$ to $1019 \mathrm{US} \$ / \mathrm{ha}\left(2.55 \mathrm{US} \$ / \mathrm{m}^{3}\right)$ for both the use of inappropriate technology in traditional harvesting and the use of the best techniques in mechanized harvesting, respectively. Relating environmental costs with the annual harvesting rate of Pinus radiata D. Don forests in Chile - at high and moderate appropriateness levels, figures of 30 to 50 million US dollars per year were obtained.

Main impacts are related to road construction, the use of prescribed fire for soil preparation practices and groundbased logging on wet soil conditions.
\end{abstract}

Key words: environmental costs, forest harvesting, sustainability, environmental assessment.

\section{RESUMEN}

Este estudio propone un método de evaluación ambiental cuantitativo, consistente en la modelación de los costos ambientales generados por las operaciones de cosecha forestal en bosques de plantación de Pinus radiata D. Don., bajo el concepto de conservación del capital natural de los recursos suelo y agua. Los costos ambientales se definen como los costos de las medidas de mitigación necesarias para bajar el nivel de impacto, hasta el grado que hubiese generado el empleo de la mejor tecnología disponible.

El modelo considera la definición de un vector de costos ambientales normalizados, los que se aplican sobre una matriz de coeficientes de impacto según la condición de fragilidad potencial de los suelos a la degradación. El resultado es una valorización de los costos ambientales por unidad de superficie que permite hacer estimaciones razonables a nivel de empresa, región y país.

Se simulan tres escenarios y niveles de apropiabilidad tecnológica diferentes. Los costos ambientales varían desde $2246 \mathrm{US} \$$ ha $\left(5.61 \mathrm{US} \$ \mathrm{~m}^{3}\right)$ hasta $1019 \mathrm{US} \$ / \mathrm{ha}\left(2.55 \mathrm{US} \$ / \mathrm{m}^{3}\right)$ para condiciones de empleo de tecnología inapropiada en cosechas semimecanizadas hasta las mejores situaciones de empleo de la técnica en cosechas mecanizadas, respectivamente. Asociando los costos ambientales a la tasa anual de corta de bosques de $P$. radiata $\mathrm{D}$. Don. en Chile, para condiciones de apropiabilidad tecnológica alta y media, se obtiene la significativa cifra de 30 a 50 millones de dólares anuales por concepto de costos ambientales relacionados al componente ambiental suelo.

Los principales impactos aparecen asociados a la construcción de caminos, el empleo de la quema como práctica de preparación de sitios para plantar y el madereo terrestre en temporada húmeda.

Palabras claves: costos ambientales, cosecha forestal, sustentabilidad, evaluación ambiental.

* Trabajo patrocinado por la Dirección de Investigación y Desarrollo de la Universidad Austral de Chile, bajo el nú- mero de registro S-95-55: "Propuesta metodológica para la evaluación de impacto ambiental en proyectos forestales". 


\section{INTRODUCCION}

La mayor parte de las actuales definiciones cualitativas de manejo sustentable de recursos naturales incluyen el mantenimiento de la productividad y el valor potencial del suelo (renta) con mínimo impacto ambiental (Goodland 1995, American Forest \& Paper Association 1995). Sin embargo, las definiciones cuantitativas generalmente no consideran la conservación del recurso natural base, que es fundamental para la sustentabilidad (Faeth 1993; Paredes 1991). Igual reflejo tiene esta situación en las cuentas nacionales, donde la mayor parte de las interacciones economíaambiente han sido ignoradas (Barker 1992). En el caso de sectores basados en capital natural, la degradación y agotamiento del ambiente natural y sus recursos contribuyen a mostrar un ingreso ficticio que no puede sostenerse en el largo plazo (Bartelmus 1989).

Routledge (1987), basado en el método estándar de Faustmann para optimizar el valor potencial del suelo de un número infinito de rotaciones forestales, asumiendo que la calidad del sitio permanece constante, desarrolló un algoritmo de programación dinámica que permite la evaluación directa de pérdidas de largo plazo asociadas con prácticas dañinas de madereo. De manera similar, pero empleando antecedentes sobre pérdida de crecimiento en plantaciones de pino radiata, Iroumé y Gayoso (1992) incorporan el efecto de la potencial degradación del sitio producida por la compactación de los suelos y su efecto en el valor neto presente de los ingresos esperados para sucesivas rotaciones. Otras dos propuestas metodológicas interesantes para el sector agrario incluyen un cálculo de las consecuencias económicas de la compactación y un cálculo del valor de depreciación del suelo (Oskoui y Voorhees 1991, Faeth 1993).

Hoy se discute que la contabilización del capital real en el caso de sectores basados en recursos naturales debiera considerar la disminución de stocks y los costos ambientales generados (Barker 1992). Pero la problemática radica en la capacidad de valorar el capital natural y costear los impactos? dado que son bienes/males que no se transan en el mercado, tienen precio pequeño o son inciertos. Como consecuencia se han desarrollado numerosos métodos directos e indirectos para obtener el valor de uso, el valor de opción y el de existencia: método del costo de viaje, valoración contingente, precios hedónicos, pérdida de productividad.

Varios métodos indirectos parecen aptos para estimar los costos ambientales según sea la situación analizada: a través del costo de las medidas mitigadoras que disminuyen los impactos (ex-después), el mayor costo de tecnologías mejoradas no alterantes (ex-antes) o simplemente el costo de oportunidad por la renta del suelo. Los mayores o menores costos dependerán, entre otros, de la apropiabilidad de la técnica y los niveles de tolerancia de los impactos. Incorporar estos costos ambientales requiere de una aceptación de conceptos de sustentabilidad, criterios e indicadores de medición, y reconocer que las tecnologías actualmente en uso generan diferentes grados de impacto al ambiente.

En Chile, el Banco Central ha iniciado un análisis de las cuentas nacionales en esta nueva concepción ambiental y deberá generar los mecanismos que permitan incorporar los costos ambientales y rebaja de stocks, como una manera de responder a la interrogante actual respecto de cuál es la tasa real de crecimiento económico del país (CONAF 1995, Hoffmann 1995).

En esta perspectiva, este estudio ofrece un modelo para valorizar monetariamente costos ambientales generados por las operaciones de cosecha forestal en plantaciones de $P$. radiata. Además, permite identificar las prácticas que generan los principales costos ambientales y posibilita el cálculo a nivel de empresa, región y país.

El modelo, aun cuando aplicable, no evalúa todos los impactos de importancia ni los beneficios ambientales, lo que se espera incorporar en etapas siguientes. Este es el caso, por ejemplo, de la influencia de las plantaciones monoespecíficas y la intervención humana sobre la diversidad biológica en relación con sistemas naturales sustituidos (Armesto et al. 1992, Franklin y Armesto 1996); efectos, que pueden ser valorados monetariamente en forma directa a través de valoración contingente o indirecta por el costo de medidas de resguardo de la biodiversidad, tales como ampliar las franjas de protección de cauces, limitar la sustitución, asegurar corredores de hábitat para conectividad y migración de especies.

Este estudio se limita sólo a los componentes ambientales suelo y agua, bajo el concepto de conservación del capital natural en el largo plazo. El 
análisis se realiza para una situación simulada de tres escenarios prototipos que emplean una técnica inapropiada, una técnica semimecanizada comparable con la apropiabilidad media del vector tecnológico nacional de los años 90 y otra mecanizada correspondiente con la mejor práctica actual. Los costos globalizados del sector se determinan a partir de las estadísticas anuales del consumo industrial de madera, asumiendo su equivalencia con el volumen cosechado.

\section{FUNDAMENTOS DE LA PROPUESTA METODOLOGICA}

Las actividades del sector forestal productivo, especialmente las operaciones de cosecha forestal, generan efectos de diferente tipo y magnitud sobre distintas variables ambientales: directos sobre el área cosechada e indirectos fuera del sitio (Gayoso 1995). Los impactos negativos serán más severos mientras más frágiles los suelos en los que se cosecha y menos apropiada la técnica empleada. Si bien se reconocen otros impactos, como efectos sobre la diversidad de especies de fauna y flora, paisaje y el componente socioeconómico y sociocultural, su valoración no se ha considerado en este estudio debido a la complejidad de los métodos de valoración requeridos (Gayoso et al. 1994). Siendo el suelo la base de la sustentabilidad del recurso forestal, se da especial énfasis a los impactos sobre este componente ambiental (Hartwig 1994).

Los costos ambientales se definen como los costos de las medidas de mitigación necesarias para bajar el nivel de impacto, hasta el nivel que hubiese generado el empleo de la mejor tecnología disponible; es decir, se acepta un cierto nivel de tolerancia de impacto que no considera costo ambiental. Así la determinación de los costos se hace por el costo de las medidas mitigadoras cuando éstas son técnicamente viables y por la pérdida de productividad de los sitios o costo de oportunidad cuando el daño se estima no recuperable o resulta impreciso costear la medida.

La definición del nivel de impacto tolerable no es sencillo de determinar en las prácticas forestales, porque generalmente no son aplicables las normas sobre emisiones, no existen normas específicas, indicadores o valores umbrales que lo regulen. En diferentes países se han elaborado códigos consistentes en la proposición de pautas para el desarrollo de las operaciones de cosecha forestal tanto para bosques naturales como de plantaciones, con la finalidad de promover prácticas de cosecha que mejoren estándares de utilización, reduzcan impactos ambientales y aseguren su manejo sustentable (Fiji, Ministry of Forests 1990, Vaughan 1990, Smith 1994, Kimmins 1993). Estas pautas entre otros consideran: la planificación de las operaciones de cosecha, la construcción de caminos y canchas de acopio, la cosecha propiamente tal (tala, desrame, trozado, madereo y acopio), el carguío y transporte, el manejo de desechos y la fuerza de trabajo, condiciones de vida y seguridad en las faenas. Sin embargo, son escasas las referencias a indicadores cuantitativos que regulen o fijen valores umbrales para los impactos tolerables producto de la cosecha forestal (British Columbia, Ministry of Forests 1991). La mayor parte de ellos se basa en listas de chequeo o análisis tipo multicriterio con gran grado de subjetividad.

A nivel nacional no existe aún este tipo de códigos, aunque ya existe discusión en esferas de universidades, Corporación Nacional Forestal (CONAF), Corporación Chilena de la Madera (CORMA), el Grupo de Producción Forestal de Fundación Chile (GPF), y algunas empresas en particular. Un ejemplo es el proyecto en desarrollo OIT-CORMA sobre medio ambiente y mundo del trabajo, que considera una propuesta en este sentido (Poschen 1994). También el GPF ha desarrollado en los últimos dos años un trabajo sostenido tendiente a promover el desarrollo de sistemas de gestión ambiental en las empresas forestales y a preparar pautas para mejorar las prácticas forestales.

Los costos de las medidas de mitigación y la información sobre la intensidad de las medidas empleadas, apropiabilidad de la técnica, distribución de las áreas y volúmenes cosechados según fragilidad de suelos, costos de técnicas mejoradas, se obtuvieron a partir de estadísticas, revisión de publicaciones, informes técnicos de empresas y comunicaciones personales (Alarcón 1990, Eeronheimo y Mäkinen 1995, Forestal Mininco S.A. 1987, INFOR 1995).

\section{EL MODELO}

El modelo calcula el costo ambiental por unidad de superticie cosechada o unidad de producto 
para la especie pino radiata. Consiste en el producto de un vector de costos normalizados $\left(\mathrm{M}_{\mathrm{i}}\right)$ sobre una matriz de coeficientes de impacto o emisiones $\left(\mathrm{E}_{\mathrm{if}}\right)$.

Los costos normalizados comprenden trece costos correspondientes a los costos de mitigación o disminución de la renta del suelo, por: 1) la pérdida de producto por las áreas destinadas a protección de cauces, 2) el exceso de superficie destinada a cortafuegos, 3) la pérdida de producto o los costos de desactivación correspondientes al área de la red mínima de caminos, 4) el área del exceso de caminos por sobre el mínimo, 5) el área ocupada por las canchas, 6) el excedente de compactación expresado como área equivalente, 7) medidas de mitigación para el control de sedimentos, 8) mantenimiento de caminos, 9) subsolado o preparación de sitios, 10) fertilización por extracción de biomasa, 11) pérdida de nutrientes por quema, 12) pérdida de nutrientes por erosión, y 13) por los costos de estabilización de taludes para mitigar la erosión en los caminos. El modelo toma la siguiente notación:

$$
C A_{f}=\sum_{i=1}^{13} \delta_{i} M_{i} E_{i f} \quad \forall f
$$

donde:

$C A_{f}=$ costo ambiental por unidad de superficie según nivel de fragilidad (f) del sitio, US\$/ha

$M_{i} \quad=$ costos de la acción mitigadora $\mathrm{i}, \mathrm{US} \$ \mathrm{ha}$

$f=$ vector fragilidad del sitio, $1=$ baja; 2=media; $3=$ alta; 4=muy alta

$\delta_{i}=$ factor de capitalización y actualización

$i=$ vector acciones de mitigación, $\mathrm{l}=$ protección de cauces, 2=desactivación cortafuegos, 3=desactivar caminos mínimos, 4=desactivar exceso caminos, $5=$ desactivar canchas, $6=$ mitigación excedente compactación, $7=$ control de sedimentos, $8=$ mantenimiento caminos, $9=$ subsolado, $10=$ reposición salida de nutrientes por extracción biomasa, 11=reposición salida de nutrientes por quema, $12=$ reposición salida de nutrientes por erosión, 13=estabilización de taludes

$E_{\mathrm{i}_{f}}=$ matriz de unidades físicas de emisión por mitigar según fragilidad del sitio, unidades/ha

Cada costo normalizado se determina a partir de información particular para cada tratamiento de mitigación y se capitaliza y actualiza para una serie infinita de rotaciones, manteniendo como supuesto un vector tecnológico constante. Esto es similar a cuando se determina el valor potencial del suelo forestal bajo ciertas restricciones (Gayoso e Iroumé 1991a, Iroumé y Gayoso 1992, Routledge 1987). Por ejemplo, el costo ambiental de no mantener franjas de protección en los cauces se determina multiplicando el área comprometida por la cantidad y el precio de los diferentes productos potencialmente cosechables.

Los costos ambientales (CA) anuales para una empresa estarán determinados por la cantidad de superficie que interviene anualmente. Los $C A$ por unidad de superficie también pueden expresarse en función del producto, dividiendo este valor por el volumen medio cosechado por unidad de superficie. Esta última expresión permite estimar los $C A$ generados por la cosecha de plantaciones de $P$. radiata a nivel nacional, multiplicando por el consumo anual de esta especie.

\section{MEDIDAS DE MITIGACION Y TOLERAN- CIA DE IMPACTOS}

En los párrafos siguientes se detallan las principales medidas mitigadoras que generan costo ambiental y se discute la mejor técnica actual.

\subsection{MANTENIMIENTO DE FRANJAS DE PROTECCION} DE CAUCES. Actualmente es de amplia aceptación dejar franjas de protección a ambos lados de los cauces de agua permanentes en el caso de bosques de plantación, constituyéndose en lo que se define como la mejor técnica. Estas franjas cumplen múltiples propósitos: atrapar sedimentos, regular la temperatura de las aguas, conectar corredores o hábitats de fauna (vertebrados, entomofauna, ictiofauna, fauna bentónica), servir de reserva de diversidad, etc. El costo ambiental desde el punto de vista de la sociedad ocurre cuando no se deja la franja de protección y su valor sería equivalente al valor de restaurar similar vegetación en igual estado de desarrollo o en su defecto el costo de oportunidad. En este último caso el costo se estima a partir del valor potencial del suelo de la superficie ocupada por las franjas. El ancho de las franjas se asocia comúnmente con el nivel de importancia del cauce y la magnitud de las pendientes de las laderas adyacentes. Para el análisis se consideró un ancho mínimo de 20 a $45 \mathrm{~m}$ a cada lado del cauce según la fragilidad potencial del sitio y que, por la longitud de los cauces por hectárea, comprende una protección de 2 a $18 \%$ de la superficie total. 
4.2. COMPENSACION POR EXCESO DE SUPERFICIE DE CORTAFUEGOS. Se estima que el requerimiento de cortafuegos alcanza de 5 a $10 \%$ de la superficie plantada (Julio 1994). Sin embargo, muchos de los caminos pueden considerarse que cumplen también la función de cortafuegos. Por lo tanto se considera como impacto ambiental sólo el exceso de superficie destinada a cortafuegos por sobre el porcentaje aportado por los caminos y que se pierde como superficie productiva. Esto a pesar del rol de protección y disminución del riesgo que significa una mayor cantidad de superficie de cortafuegos.

\subsection{CONTROL DE SEDIMENTOS. Costos indirec-} tos. Entre los costos fuera del sitio de corta es importante el generado por la erosión y sus consecuencias en la calidad de las aguas. El costo ambiental del impacto dependerá del valor económico del agua, siendo mayor en aquellos lugares donde es alto. Esto puede ser por escasez o bien por el tipo de uso que se da al agua. Igualmente las exigencias de calidad varían si se trata de aguas para consumo de la población, para riego o si son recreacionales. Bajo esta consideración Faeth (1993) hace referencias a costos del daño por tonelada de erosión entre 8.16 y $0.66 \mathrm{US} \$$ /ton para el noreste americano. La misma referencia indica para rotaciones agrícolas tradicionales (maíz-soya) en Pennsylvania, costos indirectos de 113.58 US\$/ ha/año por impactos a usuarios aguas abajo, sedimentación e impactos a recreación y pesca.

Existe gran disparidad de cifras en los estudios nacionales en cuanto a la pérdida de suelos generada por procesos de cosecha forestal y preparación de sitios. Esto se debe a que no siempre son comparables debido al diferente tamaño de las parcelas y diferentes condiciones climáticas de los años medidos. Por ejemplo, Peña et al. (1993) obtienen valores de 3.12 ton/ha/año en suelos arcillosos, para bosque cosechado con skidder y luego de quemado el residuo, en parcelas de 20 metros de longitud y $8 \%$ de pendiente. El mismo autor estima probable que las pérdidas de suelo puedan alcanzar entre 55 y 120 ton/ha/año en situaciones de laderas de 25 a $30 \%$ y gran longitud en el año peak siguiente a la tala rasa. Adicionalmente los caminos forestales aportan pérdidas de suelo; según Fahey y Coker (1989) éstas pueden alcanzar valores anuales de 1.6 a $11 \mathrm{~kg}$ por metro cuadrado de superficie de camino; esto significa $1.6 \mathrm{ton} / \mathrm{ha} /$ año para intensidades de caminos de $30 \mathrm{~m} / \mathrm{ha}$.
4.4. MITIGACION EN CAMINOS Y CANCHAS. La construcción de caminos y canchas para la cosecha genera múltiples impactos sobre los componentes ambientales suelo y agua (Gayoso et al. 1994, Gayoso 1995). Para efectos del presente análisis se identificaron las siguientes medidas mitigadoras y costos:

- medidas mitigadoras tendientes a controlar la erosión y producción de sedimentos en los taludes de corte y derrame de los caminos

- el mantenimiento de los caminos para resguardar su serviciabilidad y la construcción de obras complementarias de drenaje; por ejemplo, los pozos de decantación y disipadores de energía en la entrada y salida de alcantarillas.

- desactivación de la superficie ocupada por los caminos y las canchas.

Protección de taludes. Este ítem se calcula en base al costo de estabilización de taludes por tratamiento de hidrosiembra, considerando una superficie tratada por metro lineal de camino, determinada por la geometría del talud.

Mantenimiento de caminos. Considerando que el mantenimiento de los caminos es esencial para asegurar su estabilidad, proteger la inversión y mantener bajas tasas de erosión, se considera costo ambiental la no práctica del mantenimiento. La estimación del costo se basa asumiendo que el costo de la carpeta de rodado y obras de drenaje debe amortizarse en 25 años, o lo que es lo mismo, el costo de mantenimiento debe alcanzar al $4 \%$ anual de la inversión para mantener su serviciabilidad en el largo plazo. El gasto anual se capitaliza y actualiza en una serie infinita. La mejor tecnología comprende el mantenimiento de todos los caminos.

Desactivación exceso de caminos. La tendencia actual es la disminución de la cantidad de caminos, tanto por el problema ambiental como por los menores requerimientos de las faenas mecanizadas. Sin embargo, es común observar aún densidades camineras de $50 \mathrm{~m} / \mathrm{ha}$. Para efecto del análisis se considera costo ambiental el costo de desactivar la superficie ocupada por el exceso de caminos sobre una red mínima de $20 \mathrm{~m} / \mathrm{ha}$; esto es, los costos de subsolado, fertilización y excedente de compactación. Otra forma de cálculo es transformar esta superficie en pérdida de producto y correspondiente ingreso. La elección de uno u otro procedimiento depende del valor potencial del suelo, eligiéndose la opción de costo menor. 
Desactivación red mínima de caminos. En el análisis se consideró costo ambiental por este concepto al igual que el exceso de caminos. Sin embargo, esto puede ser discutible dado que la tecnología apropiada no debiera generar costo ambiental. Este costo se calcula a partir de la rotación siguiente a la cosecha.

Desactivación de la superficie ocupada por canchas. De forma similar a lo planteado para los caminos se valoriza como costo ambiental el costo de desactivar (subsolado, fertilización excedente de compactación) o alternativamente la pérdida de producto, en las rotaciones siguientes, por la superficie ocupada por las canchas. La mejor técnica recomienda la desactivación.

4.5. MITIGACION DE LA COMPACTACION ORIGINADA POR LA COSECHA. Existen dos formas de mirar el problema, una, evitar la compactación del suelo a través del empleo de maquinaria especial no compactante, rodados especiales de baja presión al suelo, cosechar sólo en la temporada seca; otra, volviendo a soltar el suelo. Ambas generan costos adicionales que se representan como costos ambientales. La mejor técnica es no alterar el suelo, pero no hay antecedentes de costos para esta maquinaria especial (walking machines). A nivel nacional está generalizada la segunda práctica, generalmente recuperación de los suelos por subsolados y otras técnicas culturales.

Subsolado. Esta medida mitigadora es parte de la preparación del sitio para plantar y considera el subsolado de suelos para contrarrestar la compactación producida por el tránsito de las máquinas de cosecha y favorecer la infiltración del agua. La tecnología más apropiada incluye maquinaria de diferente tipo según la topografía y grado de alteración, con una efectividad que logre recuperar la estructura y productividad perdida del suelo en un 50 por ciento (Moehring 1970). Existe costo ambiental cuando no se hace la medida mitigadora. Complementan esta medida, según sea la pendiente, terrazas y franjas de infiltración para controlar en parte el proceso erosivo. La medida mitigadora se expresa en función de las horas máquinas requeridas para subsolar una hectárea.

Excedente de compactación. Considera el excedente de compactación generado por la cosecha y que las medidas mitigadoras no logran corregir, efecto remanente que se estima en $50 \%$ del total de la pérdida de productividad del sitio (Moehring 1970). Según estudios nacionales, se ha medido que el efecto de compactación generado por el madereo puede reducir hasta un $16 \%$ el índice de sitio en la rotación siguiente y así sucesivamente (Gayoso et al. 1991, Gayoso e Iroumé 1993). El costo ambiental se obtiene en base al menor ingreso capitalizado y actualizado.

4.6. MITIGACION DE LA PERDIDA DE NUTRIENTES. La salida de elementos nutritivos se produce principalmente por la extracción de biomasa y erosión, afectando la productividad del sitio. Para que no exista pérdida de productividad, el sistema suelo-clima-planta debe alcanzar el mismo nivel de nutrientes que existía antes de la cosecha en la primera rotación, considerando así la disponibilidad de ellos para la siguiente rotación (CONAF 1995). Triviño (1993) para el caso de plantaciones de eucalipto y Alarcón (1990) citando a Toro y Alvarez (1985) para plantaciones de pino insigne, señalan que el $70 \%$ de los nutrientes almacenados en la biomasa de los árboles están contenidos en las hojas, ramas y corteza. Esto significa que el impacto ambiental dependerá de la intensidad de la extracción y tratamiento de los desechos. Si se extraen sólo los fustes y se ordenan los desechos, la extracción de los nutrientes alcanzará al 30\% del total extraído y será total en el caso de quema no controlada de los desechos. La medida mitigadora es la fertilización que considera la reposición de los elementos extraídos, valorados a precio de mercado; es costo ambiental cuando no se fertiliza en la cantidad requerida.

Se consideró además un adicional de pérdida por erosión en el caso que no practicar manejo de desechos, por ejemplo arrumado en fajas siguiendo las curvas de nivel y franjas de infiltración.

\section{COSTOS AMBIENTALES Y APROPIABILIDAD TECNICA}

La oferta de las técnicas posibles para realizar las múltiples operaciones forestales es de amplio espectro, lo que genera los más diversos tipos y niveles de impactos al cruzarlos con los tipos de fragilidad del ecositio. Esto hace muy difícil definir un vector tecnológico único representativo que permita definir la medida mitigadora requerida. El vector tecnológico se refiere al conjunto de técnicas y equipos empleados en las operaciones forestales, para ciertas condiciones y momento dado, con fines de producción a escala industrial. Por ejemplo: técnicas de preparación de sitios, si la 
empresa quema o no quema los desechos, maquinaria para cortar, apilar y arrastrar trozas, nivel de planificación de las labores de cosecha, elementos de diseño e intensidad de caminos, si se respetan franjas de protección de cauces, si se implementan medidas mitigadoras de impacto, etc.

El nivel de apropiabilidad varía en toda la gama, desde baja dando origen a los mayores costos, hasta alta donde los costos ambientales tienden a cero.

El método se aplica para los siguientes tres escenarios tecnológicos prototipos y correspondientes grados de apropiabilidad de la técnica a nivel de empresa:

a) Escenario 1: asume una práctica de cosecha de tipo tradicional con una intensificación del empleo de tractores arrastradores que dominó en Chile en los ochenta. Se otorga baja importancia a la protección de cauces, la mayor parte de bosques cosechados son aún bosques no manejados, se intensifican las redes de caminos, se aplica sólo fertilización mínima en algunos sitios para corregir deficiencias específicas como las de boro, se hace uso extensivo de la quema como práctica de preparación de sitios, se extienden las operaciones de cosecha a la temporada de invierno, se incorporan terrenos en pendientes fuertes. Para la simulación se considera la aplicación de técnicas inapropiadas y la ausencia de medidas mitigadoras.

b) Escenario 2: madereo terrestre dominado por tractores arrastradores, excepto en terrenos con pendientes sobre el $30 \%$ donde se incorporan las torres, se mejoran las técnicas de diseño y construcción de caminos (mantenimiento y estabilización de caminos), disminuye un $10 \%$ el empleo de la quema y se protegen algunos cauces principales, se practica subsolado en un $25 \%$ de terrenos.

c) Escenario 3: ingreso de la alta mecanización (cosechadoras, cabezales cortadores, tractores autocargables), alta preocupación por incorporar mejores prácticas de manejo, sensibilización de la problemática ambiental, aumento de la productividad y condiciones de vida y seguridad del trabajador forestal, masificación e intensificación de la fertilización, mayor empleo de técnicas de subsolado, manejo de residuos en un $25 \%$ y suspensión de las quemas en un $50 \%$ de las superficies, se dejan franjas de protección en todos los cauces permanentes, se disminuyen las intensidades de caminos de 50 a $30 \mathrm{~m} / \mathrm{ha}$, se desactivan canchas, etc.

El cuadro 1 resume los coeficientes de impacto, en cierta forma "emisiones contaminantes" para los tres escenarios definidos y 13 acciones de mitigación. A mayor fragilidad o susceptibilidad a la degradación del sitio se aprecia un mayor impacto ambiental para una misma tecnología de cosecha y consecuentemente se tendrán mayores costos de mitigación o costo ambiental. Lo contrario, a mayor apropiabilidad de la tecnología empleada menor será el impacto ambiental y consecuentemente menor su costo ambiental. La apropiabilidad en este estudio se entiende como la medida de qué tan bien se ajusta la técnica empleada con el medio ambiente natural, aun cuando su significado debiera alcanzar también los ámbitos económico, legal, político, social y cultural (Mustanoja 1986). En este caso, los valores de la matriz si bien preliminares representan la mejor estimación para los antecedentes disponibles; a futuro para tener una mayor certeza al evaluar a una empresa o región determinada, bastará con ingresar la información correspondiente. En todo caso se trata de datos que en su mayoría debieran estar disponibles a nivel de empresa.

Así, para calcular los costos ambientales se completa la matriz de coeficientes y se multiplica por el vector de costos normalizados y los porcentajes de distribución de superficie cosechada por nivel de fragilidad (cuadro 2). Debido a la falta de información calificada sobre la distribución de las cosechas según la fragilidad potencial de los terrenos a la degradación, excepto para algunos proyectos específicos (Gayoso 1993, Gayoso et al. 1994), para determinar la condición de cada escenario se recurre a antecedentes bibliográficos sobre niveles de erosión (Peña y Carrasco 1993, IREN 1979) y una tipificación de la superficie ocupada por plantaciones en niveles de erosión (Uriarte 1994). Se asume que si una plantación ocupa suelos que fueron clasificados en un determinado nivel de erosión, ésta estaría latente, y que al cosechar el bosque a tala rasa éste recuperaría su nivel de riesgo potencial a erosión. Se asociaron entonces los niveles de erosión de leve, moderada, grave y muy grave con los niveles de fragilidad potencial a la degradación de bajo a muy alto.

Como existen otros riesgos de degradación (remoción, deslizamientos, compactación) se estima que una clasificación más realista podría llevar a los terrenos cosechados hacia el lado de las fragilidades más altas, basta ver procesos de cosecha en temporada de invierno para comprender esta aseveración. Esta distribución toma en esta simu- 


\begin{tabular}{|c|c|c|c|c|c|c|c|c|c|c|c|c|c|}
\hline \multirow[t]{2}{*}{ Medida de mitigación } & \multicolumn{4}{|c|}{$\begin{array}{c}\text { Escenario } 1 \\
\text { Fragilidad }\end{array}$} & \multicolumn{4}{|c|}{$\begin{array}{c}\text { Escenario } 2 \\
\text { Fragilidad }\end{array}$} & \multicolumn{4}{|c|}{$\begin{array}{c}\text { Escenario } 3 \\
\text { Fragilidad }\end{array}$} & \multirow{2}{*}{$\begin{array}{l}\text { Unidad } \\
\text { por ha }\end{array}$} \\
\hline & Bajo & Medio & Alto & Muy alto & Bajo & Medio & Alto & Muy alto & Bajo & Medio & Alto & Muy alto & \\
\hline Protección de cauces & 200.0 & 400.0 & 900.0 & 1.800 .0 & 100.0 & 200.0 & 450.0 & 900.0 & 0.0 & 0.0 & 0.0 & 0.0 & $\mathrm{~m}^{2} / \mathrm{ha}$ \\
\hline $\begin{array}{l}\text { Desactivación exceso } \\
\text { cortafuegos }\end{array}$ & 100.0 & 100.0 & 100.0 & 100.0 & 100.0 & 100.0 & 100.0 & 100.0 & 100.0 & 100.0 & 100.0 & 100.0 & $\mathrm{~m}^{2} / \mathrm{ha}$ \\
\hline Control de sedimentos & 1.9 & 2.5 & 4.0 & 5.5 & 1.9 & 2.5 & 4.0 & 5.5 & 1.0 & 1.3 & 2.0 & 2.8 & ton/ha \\
\hline \multicolumn{14}{|l|}{ Mitigación en caminos y canchas } \\
\hline mantenimiento & 50.0 & 50.0 & 50.0 & 50.0 & 37.5 & 37.5 & 37.5 & 37.5 & 12.5 & 12.5 & 12.5 & 12.5 & $\mathrm{~m}^{2} / \mathrm{ha}$ \\
\hline desactivación superficie mínima & 240.0 & 256.0 & 272.0 & 288.0 & 240.0 & 256.0 & 272.0 & 288.0 & 240.0 & 256.0 & 272.0 & 288.0 & $\mathrm{~m}^{2} / \mathrm{ha}$ \\
\hline desactivación exceso camino & 360.0 & 384.0 & 408.0 & 432.0 & 360.0 & 384.0 & 408.0 & 432.0 & 156.0 & 166.0 & 177.0 & 187.0 & $\mathrm{~m}^{2} / \mathrm{ha}$ \\
\hline desactivación cancha & 200.0 & 200.0 & 200.0 & 200.0 & 200.0 & 200.0 & 200.0 & 200.0 & 0.0 & 0.0 & 0.0 & 0.0 & $\mathrm{~m}^{2} / \mathrm{ha}$ \\
\hline \multicolumn{14}{|l|}{ Mitigación por compactación } \\
\hline subsolado, casilla, terraza & 2.0 & 2.0 & 3.0 & 4.0 & 1.5 & 1.5 & 2.3 & 3.0 & 1.0 & 1.0 & 1.5 & 2.0 & h/ha \\
\hline excedente compactación & 50.0 & 50.0 & 100.0 & 100.0 & 50.0 & 50.0 & 100.0 & 100.0 & 50.0 & 50.0 & 100.0 & 100.0 & $\%$ \\
\hline \multicolumn{14}{|l|}{ Fertilización } \\
\hline control de erosión & 25.0 & 50.0 & 75.0 & 100.0 & 25.0 & 50.0 & 75.0 & 100.0 & 18.8 & 37.5 & 56.3 & 75.0 & $\%$ \\
\hline por extracción & 100.0 & 100.0 & 100.0 & 100.0 & 75.0 & 80.0 & 85.0 & 90.0 & 40.0 & 40.0 & 40.0 & 40.0 & $\%$ \\
\hline por quema & 100.0 & 100.0 & 100.0 & 100.0 & 75.0 & 80.0 & 85.0 & 90.0 & 50.0 & 50.0 & 50.0 & 50.0 & $\%$ \\
\hline
\end{tabular}


Costos ambientales. Vector de costos normalizados de las medidas mitigadoras en US\$/unidad para tres escenarios tecnológicos.

Environmental costs. Standard costs matrix of mitigation actions for three technological scenarios. US\$/unit.

\begin{tabular}{|c|c|c|c|c|c|c|c|c|}
\hline \multirow[t]{2}{*}{ Medidas de mitigación } & \multirow{2}{*}{\multicolumn{2}{|c|}{$\begin{array}{l}\text { Costos unitarios } \\
\text { normalizados }\end{array}$}} & \multicolumn{2}{|c|}{ Escenario 1} & \multicolumn{2}{|c|}{ Escenario 2} & \multicolumn{2}{|c|}{ Escenario 3} \\
\hline & & & US\$/ha & $\%$ & US\$/ha & $\%$ & US\$/ha & $\%$ \\
\hline Protección de cauces & 0.98 & $\mathrm{US} \$ / \mathrm{m}^{2}$ & 651.90 & 29.03 & 325.95 & 18.70 & 0.00 & 0.00 \\
\hline Desactivación exceso cortafuegos & 1.85 & $\mathrm{US} \$ / \mathrm{m}^{2}$ & 185.33 & 8.25 & 185.33 & 10.63 & 185.33 & 18.19 \\
\hline Control de sedimentos & 5.50 & US\$/ton & 17.62 & 0.78 & 17.62 & 1.01 & 8.81 & 0.86 \\
\hline Mitigación en caminos y canchas & & & & 30.44 & & 35.63 & & 42.24 \\
\hline protección taludes & 1.13 & $\mathrm{US} \$ / \mathrm{m}^{2}$ & 300.58 & & 300.58 & & 300.58 & \\
\hline mantenimiento & 5.00 & $\mathrm{US} \$ / \mathrm{m}$ & 250.00 & & 187.50 & & 62.50 & \\
\hline desactivación superficie mínima & 0.16 & $\mathrm{US} \$ / \mathrm{m}^{2}$ & 40.81 & & 40.81 & & 40.81 & \\
\hline desactivación exceso camino & 0.16 & $\mathrm{US} \$ / \mathrm{m}^{2}$ & 61.22 & & 61.22 & & 26.51 & \\
\hline desactivación cancha & 0.16 & $\mathrm{US} \$ / \mathrm{m}^{2}$ & 31.10 & & 31.10 & & 0.00 & \\
\hline Mitigación compactación y erosión & & & & 10.07 & & & & 15.48 \\
\hline subsolado, casilla, terraza & 54.50 & $\mathrm{US} \$ / \mathrm{h}$ & 137.01 & & 102.76 & 11.01 & 68.51 & \\
\hline excedente compactación & 124.00 & US\$/ha & 89.16 & & 89.16 & & 89.16 & \\
\hline Fertilización & & & & 21.43 & & 23.03 & & 23.23 \\
\hline por extracción & 133.00 & US\$/ha & 133.00 & & 109.08 & & 53.20 & \\
\hline por quema & 311.00 & US\$/ha & 311.00 & & 255.07 & & 155.50 & \\
\hline control de erosión & 62.00 & US\$/ha & 37.25 & & 37.25 & & 27.93 & \\
\hline Total US\$/ha & & & 2245.97 & $100 \%$ & 1743.42 & $100 \%$ & 1018.84 & $100 \%$ \\
\hline Total US $\$ / \mathrm{m}^{3}$ (1) & & & 5.61 & & 4.36 & & 2.55 & \\
\hline
\end{tabular}

(1) Considera un volumen de cosecha de $400 \mathrm{~m}^{3} / \mathrm{ha}$

lación los siguientes valores: $11.1 \%$ baja, $45.1 \%$ media, $36.2 \%$ alta y $7.6 \%$ muy alta fragilidad.

El Escenario 1, aunque representa una condición inapropiada extrema fue una condición común en los años 80 y aún es observable en la actualidad en empresas pequeñas y medianas. Para esta situación la cosecha de sucesivas rotaciones de bosques de $P$. radiata cada 22 años puede generar costos ambientales equivalentes a 2.246 US\$/ha, lo que llevado a una producción media de $400 \mathrm{~m}^{3} / \mathrm{ha}$ se transforma en $5.61 \mathrm{USS} / \mathrm{m}^{3}$. Este valor se considera de una gran significación, por cuanto representa el $13.2 \%$ para un precio de mercado de la madera ponderado por tipo de producto de $42.58 \mathrm{US} \$ / \mathrm{m}^{3}$.

Al expresar el costo ambiental como una anualidad, para una tasa de descuento del $12 \%$, este valor es igual a $269.52 \mathrm{US} \$ / \mathrm{ha} / \mathrm{año}$, valor que es mayor que lo señalado por Faeth (1991) para cosechas agrícolas, donde contabiliza 25 US\$/acre/ año por concepto de depreciación del suelo y 46
US\$/acre/año por concepto de costos fuera del sitio. La diferencia radica en que las estimaciones de Faeth no consideran la pérdida de producto por reducción de superficies productivas, esto es, superficie de protección de cauces, caminos y cortafuegos.

Los tres mayores responsables del impacto se atribuyen a la cosecha de bosques plantados hasta las orillas de las quebradas (29\%), a la práctica de construcción de caminos llamada "bote al lado" y la no estabilización de los correspondientes taludes de derrame (30\%) y el indiscriminado empleo de la quema en la preparación de sitios para plan$\operatorname{tar}(21 \%)$.

El Escenario 2 bien podría representar las cosechas semimecanizadas de principio de los años 90, donde las mejoras tecnológicas en los procesos productivos tienen su reflejo positivo en los costos ambientales que disminuyen un $22 \%$, aunque los principales impactos persisten. 
A partir del año 1994, se percibe en Chile una efectiva disminución de los costos ambientales generados por las cosechas a nivel de las grandes empresas forestales. Esto es lo que pretende reflejar el Escenario 3, donde los costos ambientales remanentes alcanzan a 1019 US\$/ha, es decir menos de la mitad de los que origina el empleo de técnicas inapropiadas. Es seguro que la mejor planificación de las faenas, adecuadas decisiones de los supervisores y contratistas en la ejecución de las operaciones, mejor selección de maquinaria, muchas sin significar para la empresa grandes desembolsos han tenido un claro beneficio ambiental. Dos son los problemas que requieren la mayor atención dado que se llevan el $65 \%$ de los costos: a) la disminución de los niveles de remoción de suelo y erosión generados por el exceso de construcción de caminos y la no estabilización de taludes de derrame, y b) los elevados niveles de extracción de elementos nutritivos del suelo sin una compensación adecuada por fertilización. Le siguen también los costos asociados a la compactación que genera el empleo de maquinaria de cosecha de mayor tamaño.

Se debe tener en cuenta, sin embargo, que la situación que describe el Escenario 3 no es una condición generalizada actualmente en Chile. Más bien se trata de metas que han comenzado a autoimponerse algunas empresas que están preocupadas del manejo sustentable del recurso forestal, como parte de la sustentabilidad de su negocio y de la permanencia de sus productos en un mercado cada vez más exigente en materia ambiental.

Así los costos ambientales anuales generados por la cosecha de bosques de $P$. radiata, asumiendo un consumo de 13 millones de metros cúbicos de madera proveniente de tala rasa (unas 33000 ha/año), alcanzarían de 30-50 millones de dólares según se cuente con la tecnología del Escenario 3 ó 2 , respectivamente.

A nivel preliminar se puede señalar que las cosechas de bosques de eucalipto, en rotaciones de 10 años, generarían mayores costos ambientales que las cosechas de $P$. radiata, debido a que generalmente ocupan suelos con mayor valor potencial, a la mayor tasa de extracción de elementos nutritivos, a la necesidad de entrar a cosechar y transitar el suelo con la maquinaria el doble de veces, entre otros.

En cuanto al bosque nativo, existe la percepción pública que los costos ambientales de estas cosechas, especialmente aquellas donde se ha practicado tala rasa, serían mayores que los del sector plantaciones. Esto lo corroboran estudios que reconocen una reducción de la diversidad de especies de aves, pequeños mamíferos y plantas vasculares en sectores manejados y donde ha ocurrido sustitución por plantaciones monoespecíficas con especies exóticas, en relación a áreas originales de bosque nativo (Franklin y Armesto 1996). Sin embargo, no se tienen aún antecedentes de valoración económica de estos impactos.

Aun cuando puede ser discutible la extrapolación de los costos unitarios al volumen total de producción anual del sector forestal, hoy del orden de los 20.8 millones de $\mathrm{m}^{3} / \mathrm{año}$, los razonamientos anteriores conducen a que al menos es esperable un rango de costos ambientales de 53 a 91 millones de dólares anuales, valor que representa aproximadamente el 2-3\% del PIB del sector.

Los valores que entrega la simulación, si bien menos relevantes en cuanto a exactitud son la primera valoración publicada y representan un muy probable rango de magnitud. Esto debiera servir para dimensionar el problema, estudiar y discutir las posibles regulaciones y políticas que puedan ayudar a reducir los impactos hasta niveles admisibles. Sin embargo, por la complejidad de la valoración de los ecosistemas, el problema no está resuelto y se requiere aún de un esfuerzo multidisciplinario para el mejor análisis de los factores que influyen en la relación entre el ser humano y el ambiente.

\section{CONCLUSIONES}

Lo importante de este trabajo es la generación de un modelo capaz de estimar los costos ambientales a partir de información de fácil obtención, que permite, además, jerarquizar los principales impactos y actividades que le dan origen. Los vectores de costos normalizados y las matrices de coeficientes de impacto podrán ser perfeccionados según se obtenga información cualificada, ya sea modificando sus valores o incorporando o quitando variables. El método igualmente es aplicable a otras especies forestales y podrá incorporar otros impactos sobre restantes componentes ambientales en la medida que sea posible reconocer la acción de mitigación.

Siendo una propuesta metodológica que se sustenta en una base teórica, deberá someterse a una amplia discusión entre los actores empresariales, institucionales y científicos, con la finalidad de 
fundamentar la inclusión o no de éstos u otros costos y beneficios, la fijación de indicadores de sustentabilidad y valores umbrales, la aceptación o no del trueque de disminución de capital natural por capital creado ("sustituibilidad"), etc. Debe tenerse presente que es uno de los primeros esfuerzos metodológicos cuantitativos en su género, aun cuando tiene una base de comparación con modelos aplicados para calcular la depreciación del suelo en la agricultura.

\section{BIBLIOGRAFIA}

ALARCON, C. 1990. "¿Los costos ocultos en el sector forestal disminuyen la rentabilidad del negocio?", Renares 7(30): 3-14.

AMERICAN FOREST \& PAPER ASSOCIATION. 1995. Sustainable forestry; Principles and implementation guidelines. Washington, 9 pp.

ARMESTO, J., C. SMITH-RAMIREZ, P. LEON, M.K. ARROYO. 1992. "Biodiversidad y conservación del bosque templado en Chile", Ambiente y Desarrollo Vol. VIII $\mathrm{N}^{\circ} 4$ : 19-24.

BARKER, T. 1992. Accounting for the environment. Department of Applied Economics. University of Cambridge, 11 pp.

BARTELMUS, P. 1989. "Environmental accounting and the system of national accounts". En AHMAD, Yusuf. Salah EL SERAFY and Ernst LUTZ (eds.), Environmental accounting for sustainable development. The World Bank, Washington D.C., pp. 79-87.

BRITISH COLUMBIA, MINISTRY OF FOREST. 1993. Forest road and logging trail engineering practices. 19 p. Apéndices y anexos.

CONAF. 1995. Informe sobre el bosque nativo del Banco Central: Un ejercicio perfectible. Chile Forestal. Chile, 234: $8-10$.

EERONHEIMO, O., P. MÄKINEN. 1995. Desarrollo de cosecha forestal en las plantaciones de Pino radiata en Chile. Instituto de Investigaciones Forestales de Finlandia. Serie Informativa del Instituto de Investigaciones Forestales de Finlandia 542, 79 pp.

FAETH, P. 1993. "Evaluating agricultural policy of production systems: an economic framework", Journal of soil and water conservation 48(2): 94-99.

FAHEY. B., R. COKER. 1989. "Forest road erosion in the granite terrain of Southwest Nelson. New Zealand", Journal of Hydrology (N.Z.) 28(2): 123-141.

FORESTAL MININCO. 1987. Desarrollo en cosecha y transporte forestal. Comité técnico, s.p.

FIJI, MINISTRY OF FOREST. 1990. Fiji national code of logging practice, s.p.

FRANKLIN, J., J. ARMESTO. 1996. "La retención de elementos estructurales del bosque durante la cosecha: una alternativa de manejo para los bosques nativos chilenos", Ambiente y Desarrollo Vol. XII $N^{\circ}$ 2: 69-79.

GAYOSO, J. 1993. Investigación sobre efectos en el suelo y el agua de las plantaciones de E. globulus de VOLTERRA S.A. Informe Técnico, Valdivia, $53 \mathrm{pp.} \mathrm{y} \mathrm{anexos.}$

1995. Impacto de plantaciones sobre el ambiente físico. En: Actas Simposio IUFRO Para Cono Sur Sudamericano: Manejo Nutritivo de Plantaciones Forestales. Valdivia, pp. 271-283.
GAYOSO, J., A. IROUME. 1991. Impacto de la degradación de suelos en el valor neto presente esperado en plantaciones. Sin publicar.

1993. Impacto al suelo por efecto de la cosecha forestal. En: Boletín $\mathrm{N}^{\circ} 10$ Suelos Forestales. Sociedad Chilena de la Ciencia del Suelo. Valdivia, pp. 98-106.

GAYOSO, J., A. IROUME, A. ELliES. 1991. Degradación de suelos forestales asociada a operaciones de cosecha. En: Actas III Taller de Producción Forestal. Grupo de Producción Forestal. Empresas Forestales/Fundación Chile. Concepción, s.p.

GAYOSO, J., M. MENESES, A. UNDA, Y. MARTINEZ. 1994. Evaluación de impacto ambiental del proyecto de plantaciones de Eucalyptus globulus Forestal Los Lagos S.A. Universidad Austral de Chile. Informe Convenio, Valdivia, 121 pp. y anexos.

GOODLAND, R. 1995. "The concept of environmental sustainability", Аnnu. Rev. Ecol. Syst. 26: 1-124.

HARTWIG, F. 1994. La tierra que recuperamos. Editorial Los Andes. Serie medioambiente y desarrollo. Santiago, $256 \mathrm{pp}$

HOFFMANN, A. 1995. "Cuentas ambientales y bosque nativo", Rev. Vivienda y Decoración, "El Mercurio", 28 de octubre, pp. 76-77.

INFOR. 1995. Estadísticas Forestales 1994. Boletín estadístico $\mathrm{N}^{\circ} 40,113 \mathrm{pp}$.

IROUME, A., J. GAYOSO 1992. Degradación de suelos forestales y disminución del valor neto presente esperado en plantaciones. En: Pinus radiata investigación en Chile. Valdivia, pp. 231-238

IREN. 1979. Fragilidad de los ecosistemas naturales de Chile. Informe 40. Instituto Nacional de Recursos NaturalesCORFO, s.p.

JULIO, G. 1994. Fundamentos del manejo del fuego. Univ. de Chile, Fac. de Ciencias Agrarias y Forestales, Departamento de Manejo de Recursos Forestales, $267 \mathrm{pp}$.

KIMMINS, H. 1993. Balancing Act. Environmental Issues in Forestry. UBC Press. Vancouver, $244 \mathrm{pp}$.

MOEHRING, D. 1970. "Forest soil improvement through cultivation", Journal of forestry. Junio 1970: 328-331.

MUSTANOJA, K. 1986. A review of methodology for evaluation of appropriateness. Apropriate forest industries. FAO Forestry paper 68, pp. 311-324.

OSKOUI, K., W. VOORHEES. 1991. "Economic consequences of soil compaction", Transactions of the American Society of Agricultural Engineers 34(6): 2317-2323.

PAREDES, G. 1991. Economía del bosque nativo: conservación o tala rasa. CORMA $\mathrm{N}^{\circ}$ 221: 31-34.

PEÑA, L., P. CARRASCO. 1993. Clasificación preliminar de erosión en los suelos de la cuenca del río Biobío. En: Serie Propuestas de Ordenamiento Vol. 4. Seminario Internacional Elementos Cognoscitivos sobre el Recurso Suelo y Consideraciones Generales Sobre el Ordenamiento Agroforestal EULA. Ministerio Degli Affari Esteri Delia República Italiana/Ministerio de Planificación y Cooperación de la República de Chile. Concepción, pp. 33-45.

PEÑA, L. P. CARRASCO, M. FIGUEROA, C. OYARZUN, B. LO CASCIO. 1993. Pérdidas por erosión hídrica en suelos agrícolas y forestales de la cuenca del río Bío-bío. En Serie Propuestas de Ordenamiento Vol. 4. Seminario Internacional Elementos Cognoscitivos sobre el Recurso Suelo y Consideraciones Generales sobre el Ordenamiento Agroforestal. EULA. Ministerio Degli Affari Esteri Delia República Italiana/Ministerio de Planificación y Cooperación de la República de Chile. Concepción, pp. 47-60.

POSCHEN, N. 1994. Proyecto medio ambiente y Mundo de trabajo. OIT, Santiago, s.p.

ROUTLEDGE, R. 1987. "The Impact of Soil Degradation on the Expected Present Net Worth of Future Timber Harvests", Forest Science 33(4): 823-834. 
SMITH, R. 1994. British Columbia Forest Practices Code. Standards with revised rules and field guide references. Ministry of Forest. Vernon, B.C., $215 \mathrm{pp}$.

TRIVIÑO, T. 1993. "Las plantaciones: medio eficaz para la recuperación de ecosistemas degradados", CORMA 234: 44-47.
URIARTE, A. 1994. "Plantaciones en suelos erosionados y sustitución: ¿qué superficie?", CORMA 239: 35-37.

VAUGHAN, L. 1990. New Zealand Forest code of Practice. En Informe $\mathrm{N}^{\circ} 10$, Código de prácticas forestales. GPF. Concepción. Traducción, 69 pp. 